Article

\title{
A retrospective cohort study of the clinical features, pathological characteristics, and molecular classification in reproductive age patients with endometrial cancer who experienced unsuccessful fertility-sparing treatment
}

\author{
Tong Yu MD 2, Xi-Wen Zhang MD 1, Ping-Li Sun MD ${ }^{3}$, Abdulkarim Mohamed Farah MM 1, Yan Jia MD 1,* \\ 1 Department of gynecology, the second hospital of Jilin University, Changchun, Jilin Province, China; \\ 13044306827@163.com \\ 2 Department of orthopaedics, the second hospital of Jilin University, Changchun, Jilin Province, China; \\ spinesurgery@yeah.net \\ 3 Department of pathology, the second hospital of Jilin University, Changchun, Jilin Province, China; ping- \\ lisun@jlu.edu.cn \\ * Correspondence: jiayan@jlu.edu.cn
}

\begin{abstract}
:
Background: The purpose of the present study was to retrospectively evaluate clinical features, pathological characteristics, and molecular classification among patients with early-stage endometrioid cancer (EEC) in reproductive age following unsuccessful fertility-sparing treatment.

Methods: We enrolled 18 EEC patients who experienced failure of fertility-sparing treatment in our hospital from January 2011 to October 2021. Then, fertility-sparing treatment outcomes and prognosis were recorded. We compared preoperative curettage biopsy with histopathological results. In addition, we performed molecular classification of all patients using second-generation sequencing to determine which type of molecular endometrial cancer $(E C)$ was not suitable for fertility preservation therapy.
\end{abstract}

Results: Six patients showed more severe pathologic types in histopathology than in curettage biopsy results. Besides, molecular classification results showed 15 patients with CNL, 2 patients with MSI-H, and 1 case with 'unclassifiable'. All 18 patients were infertility following fertility-sparing treatment.

Conclusions: Molecular classification could provide reliable supplementary information for evaluating prognostic and contribute to treatment option decision-making in EEC patients. Fertility-sparing treatment is not recommended for EEC patients with CNH and MSI-H. Furthermore, fertilitysparing treatment can be attempted in EEC patients with CHL, but regular follow-up should be carried out to early detection of EC relapse and prevention of disease progression.

Keywords: endometrial cancer; fertility-sparing management; molecular classification; reproductive age; pregnancy

\section{Introduction}

Endometrial cancer (EC) is one of the most common invasive malignant neoplasms of the female genital tract $[1,2]$. More than $90 \%$ of EC are diagnosed in perimenopause 
women [3], and $15 \% \sim 25 \%$ in premenopausal patients [2, 4]. However, $2.1 \% \sim 14.4 \%$ of women with EC are younger than 40 years old [1,5], and over $70 \%$ of them are nulliparous at diagnosis since in the current era women delay their childbearing [3]. Moreover, EC has an increasing incidence in adolescents [6], and even in 13-year-old girl [7]. In this scenario, the standard surgical intervention of hysterectomy and bilateral salpingo-oophorectomy may not be ideal for reproductive age patients who plan to preserve their fertility [8]. Thus, fertility-sparing treatment for such patients is strongly demanded $[2,9]$.

EEC was defined as tumor grade 1, absence of extrauterine metastases, absence of lymphovascular space, myometrial, or cervical invasion [10]. Fertility-sparing treatment approaches for EEC are mainly progestin therapies, including medroxyprogesterone acetate (MPA), megestrol acetate (MA), levonorgestrel intrauterine system (LNG-IUS) [1114], and comprehensive hysteroscopic evaluation and lesion resection plus progestin therapy [15]. Successful pregnancy cases with EEC following fertility-sparing treatment have been reported worldwide [16]. However, the first difficulty of fertility-sparing treatment for some EEC patients is disease progression while on treatment or after initial response to medical treatment [2], which might lead to infertility, spontaneous abortion [15], and even life-threatening; the second difficulty was that fertility-sparing treatment, such as hormonal therapy, is a non-standard treatment method for EEC [2], and carries potential adverse outcomes. Therefore, an effective method for evaluating the prognosis of fertilitysparing treatment in young patients with EEC is essential, facilitating screening candidates for fertility-sparing treatment and reducing the risk of failure.

Traditionally, EC has been classified into two main clinic-pathological types: Type I and Type II [3]. Type I is the endometrioid type [17] because it is similar to the endometrium and is characterized by genetic predisposition (e.g., Lynch syndrome-LS); it usually has a favorable outcome due to minimal myometrium invasion [18]. Type II EC is associated with higher patient age, high stage and grade, non-endometrioid histology, and poor prognosis. Type II includes several subtypes such as serous, clear cell, and undifferentiated carcinomas [19]. However, traditional typing cannot meet current clinical needs because it cannot accurately predict patient survival and disease outcome. In 2013, a molecular classification scheme was proposed by The Cancer Genome Atlas (TCGA), which can evaluate the disease prognosis of EC [20]. However, few studies focus on molecular classification features in young patients with EEC who experienced pregnancy failure after fertility-sparing treatment. We hypothesized that molecular classification results have an association with fertility-sparing treatment outcomes in EEC patients. Herein, we retrospectively evaluated the clinical features, pathological characteristics, molecular classification, and fertility-sparing treatment outcomes in EEC patients.

\section{Results}

\subsection{Demographic characteristics of patients}

All 18 patients were initially diagnosed with G1 EC. The patient's age ranged from 26 to 40, with an average of 33.67 years. The median age of menarche was 13.56 years (1116 years). Among the 18 patients, 3 were unmarried and had no history of pregnancy; the other 15 were married, of which 10 were primarily infertile. In addition, 12 patients had irregular non-menstrual bleeding, 11 patients had irregular menstrual cycle; 3 cases had a family history of EC, 3 patients were complicated with polycystic ovary syndrome (PCOS); 8 patients (44.44\%) were obese (BMI $\geq 28 \mathrm{~kg} / \mathrm{m} 2)$, and 2 of these 8 had obesity and PCOS; only 1 patient has a history of intra-cervical epithelioid neoplasia (Grade 3) (Case 2) (Table 1). Serum CAl25 and imaging evaluation were performed preoperatively. The results of serum CAl 25 were normal in all patients. Moreover, the curettage biopsy results of all patients are shown in Table 2.

\subsection{Pregnancy outcomes after fertility-sparing treatment}

2.2.1. The patients had complete remission after fertility-sparing treatment for EC but remained infertile 
6 cases achieved complete remission of EC, among which 3 patients (case 7, 13, and 17) had no relapse and did not undergo surgery, but the other 3 cases (case 1, 9, and 11) suffered from tumor relapse and performed a hysterectomy. Unfortunately, none has yet been pregnant until now (Table 2).

\subsubsection{The patients had stable disease after fertility-sparing treatment for EC and remained infertile}

12 patients showed stable disease which did not respond to progesterone after 3-9 months of treatment, and the disease was not reversed. Among them, case 2 has a history of intra-cervical epithelioid neoplasia (Grade 3); cases 7, 9, and 16 with EC had PCOS; case 14 had liver disease; case 10 with EC has hypertension; case 1, 5, 7, 10, 13, 15, 16, and 18 with EC had obesity (BMI $\geq 28 \mathrm{~kg} / \mathrm{m} 2$ ). Moreover, the curettage biopsy was performed 3 , 6 , or 9 months after fertility-sparing treatment and suggested stable disease. The immunohistochemical (IHC) results showed a negative progesterone receptor. Thus, all the 12 EC patients, who presented with the stable disease following fertility-sparing treatment, underwent a hysterectomy.

\subsubsection{Surgical intervention, ovarian preservation, and histopathological results}

Totally 15 patients performed surgical intervention after fertility-sparing treatment due to EC recurrence (cases 1, 9, and 11), stable disease (cases 2-5, 8, 10, 12, 14-16, and 18), and progression disease (case 6) (Table 2).

12 of 15 patients who underwent surgery had ovaries preservation. Moreover, intraoperative ovarian biopsies were performed in 8 of the 12 patients with ovaries preservation, and all results were negative. In the 12 patients with ovaries preservation, 7 cases have bilateral ovaries preservation, and 5 cases have unilateral ovaries preservation.

6 of 15 patients have different pathologic types in histopathology compared to curettage biopsy results. All patients were G1 EC in curettage biopsy. However, histopathology results were Mullerian mixed sarcoma in case 2, G1G2 EC in cases 3, 6, and 10-12 (Table 2).

\subsection{Molecular classification}

Molecular typing detection showed that cases 1, 3-12, and 15-18 were CNL, cases 2 and 14 were MSI-H. The tumor concentration of case 13 samples was too low to carry out a molecular classification test, so no results were available. Moreover, the details of molecular classification are presented in Table 4.

\subsection{Follow-up}

The median follow-up time was 39 months (13 months to 120 years), none developed new neoplasms, and all patients survived at the final follow-up visit. Besides, 1 (case 2) experienced colon cancer 4 years after gynecologic surgery and then performed partial colorectal resection.

\subsection{Figures and Tables}




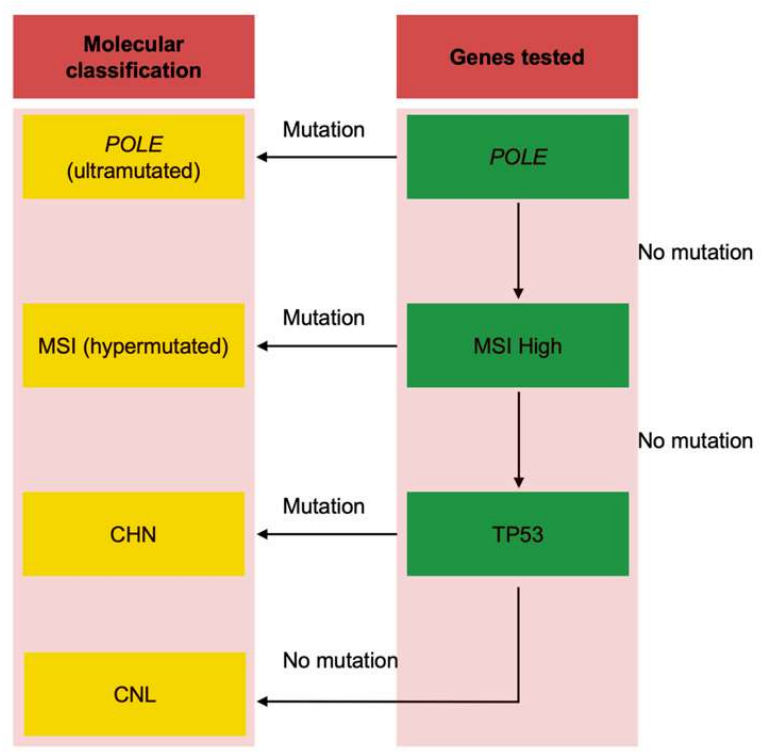

Figure 1. Method of molecular classification. $\mathrm{CHL}$, copy number abnormalities low; $\mathrm{CNH}$, copy number abnormalities high; MSI, microsatellite instability; MMR, mismatch repair.

Table 1. Demographic characteristics of patients.

\begin{tabular}{|c|c|c|c|c|c|c|c|c|c|c|c|c|}
\hline No. & MS & $\begin{array}{l}\text { Age } \\
(y r)\end{array}$ & PP & $\begin{array}{c}\text { BMI } \\
\left(\mathrm{Kg} / \mathrm{m}^{2}\right)\end{array}$ & $\begin{array}{c}\text { AAM } \\
(y r)\end{array}$ & $\begin{array}{c}\text { MMCL } \\
\text { (d) }\end{array}$ & $\begin{array}{l}\text { MPL } \\
\text { (d) }\end{array}$ & INMB & IMC & PCOS & FHEC & PI \\
\hline 1 & Yes & 40 & G0, P0 & 33.53 & 14 & 60 & 7 & Yes & Yes & No & No & Yes \\
\hline 2 & Yes & 26 & G1, P0 & 19.53 & 13 & 30 & 8 & Yes & No & No & Yes & No \\
\hline 3 & No & 29 & - & 21.64 & 13 & 60 & 7 & No & Yes & No & Yes & - \\
\hline 4 & Yes & 34 & G0, P0 & 23.03 & 14 & 30 & 8 & Yes & No & No & No & Yes \\
\hline 5 & Yes & 32 & G0, P0 & 31.24 & 13 & 60 & 15 & Yes & Yes & No & No & Yes \\
\hline 6 & Yes & 31 & G0, P0 & 21.61 & 13 & 30 & 7 & No & No & No & No & Yes \\
\hline 7 & No & 33 & - & 43.25 & 13 & 28 & 7 & Yes & No & Yes & No & - \\
\hline 8 & Yes & 33 & G0, P0 & 25.28 & 14 & 60 & 3 & No & Yes & No & No & No \\
\hline 9 & Yes & 32 & G0, P0 & 23.62 & 13 & 90 & 7 & No & Yes & Yes & No & Yes \\
\hline 10 & Yes & 38 & G3, P0 & 36.79 & 11 & 90 & 15 & Yes & Yes & No & No & No \\
\hline 11 & Yes & 27 & G0, P0 & 27.34 & 12 & 180 & 7 & Yes & Yes & No & No & Yes \\
\hline 12 & Yes & 35 & G0, P0 & 23.88 & 16 & 180 & 6 & No & Yes & No & No & Yes \\
\hline 13 & Yes & 32 & G0, P0 & 35.16 & 15 & 120 & 8 & Yes & Yes & No & No & Yes \\
\hline 14 & Yes & 33 & G4, P1 & 24.77 & 13 & 25 & 7 & Yes & No & No & No & No \\
\hline 15 & Yes & 40 & G0, P0 & 31.23 & 14 & 35 & 7 & No & No & No & No & Yes \\
\hline 16 & No & 36 & - & 33.80 & 13 & 30 & 7 & Yes & Yes & Yes & No & - \\
\hline 17 & No & 36 & - & 27.68 & 16 & 30 & 6 & Yes & No & No & No & - \\
\hline 18 & Yes & 39 & G0, P0 & 36.73 & 14 & 30 & 15 & Yes & Yes & No & Yes & Yes \\
\hline Average & - & 33.67 & - & 28.90 & 13.56 & 64.89 & 8.17 & - & - & - & - & - \\
\hline
\end{tabular}

MS, marital status; PP, previous pregnancies; G, gestation; P, parturition; -, asexual life; BMI, body mass index; AAM, age at menarche; MMCL, maximal menstrual cycle length; MPL, menstrual phase length; INMB, irregular non- 
menstrual bleeding; IMC, irregular menstrual cycle; PCOS, polycystic ovary syndrome; FHEC, family history of endometrial cancer; PI, primary infertility; yr, year; $d$, day.

Table 2. The curettage biopsy, conservative treatment approaches, treatment outcomes, surgical treatment strategies, and pathological results of patients were recorded.

\begin{tabular}{|c|c|c|c|c|c|c|c|c|c|c|c|c|c|c|}
\hline \multirow{2}{*}{ No. } & \multirow{2}{*}{ CB } & \multirow{2}{*}{ СТA } & \multirow{2}{*}{ Tо } & \multirow{2}{*}{ DT $^{*}$} & \multirow{2}{*}{ DOR $^{*}$} & \multirow{2}{*}{ STS } & \multirow{2}{*}{ PT } & \multirow{2}{*}{ Histopathology } & \multirow{2}{*}{ MI } & \multirow{2}{*}{ Stage } & \multicolumn{3}{|c|}{ IHC } & \multirow{2}{*}{$\mathrm{FU}^{*}$} \\
\hline & & & & & & & & & & & ER & PR & P53 & \\
\hline 1 & $\begin{array}{l}\text { G1 } \\
\text { EC }\end{array}$ & EEL+MPA & $C R$ & 6 & 12 & $\mathrm{LH}+\mathrm{BS}$ & None & G1 EC & NMI & IA & ++ & +++ & - & 120 \\
\hline 2 & $\begin{array}{l}\text { G1 } \\
\text { EC }\end{array}$ & MA & SD & 6 & - & TAH++BSO+PLND & TC & MMDMT & SMI & IA & ++ & - & - & 100 \\
\hline 3 & $\begin{array}{l}\text { G1 } \\
\text { EC }\end{array}$ & MPA & SD & 6 & - & TAH+BSO+PLND & TC & G1G2 EC & SMI & IA & +++ & +++ & - & 88 \\
\hline 4 & $\begin{array}{l}\text { G1 } \\
\text { EC }\end{array}$ & MPA & SD & 9 & - & $\mathrm{LH}+\mathrm{BS}$ & None & G1 EC & NMI & IA & ++ & ++ & - & 52 \\
\hline 5 & $\begin{array}{l}\text { G1 } \\
\text { EC }\end{array}$ & MPA & SD & 3 & - & $\mathrm{LH}+\mathrm{BS}$ & None & G1 EC & NMI & IA & ++ & ++ & - & 51 \\
\hline 6 & $\begin{array}{l}\text { G1 } \\
\text { EC }\end{array}$ & MPA & PD & 3 & - & $\mathrm{LH}+\mathrm{BSO}$ & None & G1G2 EC & SMI & IA & ++ & ++ & - & 50 \\
\hline 7 & $\begin{array}{l}\text { G1 } \\
\text { EC }\end{array}$ & MA & $\mathrm{CR}$ & 6 & NR & I & I & I & I & I & I & I & I & 48 \\
\hline 8 & $\begin{array}{l}\text { G1 } \\
\text { EC }\end{array}$ & MPA & SD & 3 & - & $\mathrm{TAH}+\mathrm{BS}$ & None & G1 EC & SMI & IA & ++ & ++ & - & 42 \\
\hline 9 & $\begin{array}{l}\text { G1 } \\
\text { EC }\end{array}$ & MPA & $\mathrm{CR}$ & 6 & 6 & $\mathrm{TAH}+\mathrm{BS}$ & None & G1 EC & NMI & IA & +++ & +++ & + & 40 \\
\hline 10 & $\begin{array}{l}\text { G1 } \\
\text { EC }\end{array}$ & MA/MPA & SD & $3 / 6$ & - & $\mathrm{LH}+\mathrm{BS}$ & None & G1G2 EC & NMI & IA & +++ & +++ & - & 39 \\
\hline 11 & $\begin{array}{l}\text { G1 } \\
\text { EC }\end{array}$ & MPA & $C R$ & 6 & 3 & TAH+BS+PLND & None & G1G2 EC & SMI & IA & ++ & ++ & - & 38 \\
\hline 12 & $\begin{array}{l}\text { G1 } \\
\text { EC }\end{array}$ & MPA & $\mathrm{SD}$ & 3 & - & $\mathrm{LH}+\mathrm{BS}$ & None & G1G2 EC & SMI & IA & +++ & + & - & 36 \\
\hline 13 & $\begin{array}{l}\text { G1 } \\
\text { EC }\end{array}$ & MPA & $C R$ & 6 & NR & I & I & I & I & I & I & I & I & 33 \\
\hline 14 & $\begin{array}{l}\text { G1 } \\
\text { EC }\end{array}$ & LNG-IUS & SD & 3 & - & $\mathrm{LH}+\mathrm{BS}$ & None & G1 EC & NMI & IA & + & ++ & - & 28 \\
\hline 15 & $\begin{array}{l}\text { G1 } \\
\text { EC }\end{array}$ & MPA & SD & 6 & - & TAH+BS+PLND & None & G1 EC & SMI & IA & ++ & + & - & 22 \\
\hline 16 & $\begin{array}{l}\text { G1 } \\
\text { EC }\end{array}$ & MPA & SD & 9 & - & $\mathrm{LH}+\mathrm{BS}$ & None & G1 EC & NMI & IA & +++ & +++ & - & 22 \\
\hline 17 & $\begin{array}{l}\text { G1 } \\
\text { EC }\end{array}$ & EEL+MPA & $C R$ & 6 & NR & I & I & I & I & I & I & I & I & 18 \\
\hline 18 & $\begin{array}{l}\text { G1 } \\
\text { EC }\end{array}$ & MPA & SD & 3 & 0 & $\mathrm{TAH}+\mathrm{BS}$ & None & G1 EC & SMI & IA & ++ & ++ & + & 13 \\
\hline
\end{tabular}


$\mathrm{CB}$, curettage biopsy; $\mathrm{TO}$, treatment outcome; $\mathrm{PD}$, progression disease; $\mathrm{SD}$, stable disease; CR, complete regression; CTA, conservative treatment approaches; EEL, excision of endometrial lesions; MPA, medroxyprogesterone acetate; MA, megestrol acetate; LNG-IUS, levonorgestrel intrauterine system; DT, duration of treatment; DOR, duration of overall response; NR, no relapse; STS, surgical treatment strategies; LH, laparoscopic hysterectomy; BSO, bilateral salpingooophorectomy; TAH, trans-abdominal hysterectomy; PLND, pelvic lymph node dissection; BS, bilateral salpingectomy; PT, postoperative treatment; PR, pathological results; MMDMT, malignant Mullerian duct mixed tumor; MI, myometrial invasion; NMI, no myometrial invasion; SMI, superficial muscular infiltration; G1 EC, high differentiation endometrial cancer; G1G2 EC, high-medium differentiation endometrial cancer; IHC, immunohistochemical; ER, estrogen receptor; PR, progesterone receptor; FU, follow-up; *, month.

Table 3. The genes, ranges, and types of sequencing.

\begin{tabular}{|c|c|c|}
\hline Genes & Ranges of detection & Types of detection \\
\hline POLE & Exonuclease domain, and partial region of exon 19 & \multirow{2}{*}{ Point mutation, insertion/deletion } \\
\hline TP53 & Coding area & \\
\hline MSI & 55 gene locus of MSI & \\
\hline \multicolumn{3}{|c|}{ POLE indicates polymerase epsilon, and MSI represents microsatellite instability. } \\
\hline No. & \multicolumn{2}{|c|}{ Molecular classification } \\
\hline 1 & \multicolumn{2}{|c|}{ CNL } \\
\hline 2 & \multicolumn{2}{|c|}{ MSI-H } \\
\hline 3 & \multicolumn{2}{|c|}{ CNL } \\
\hline 4 & \multicolumn{2}{|c|}{$\mathrm{CNL}$} \\
\hline 5 & \multicolumn{2}{|c|}{$\mathrm{CNL}$} \\
\hline 6 & \multicolumn{2}{|c|}{$\mathrm{CNL}$} \\
\hline 7 & \multicolumn{2}{|c|}{$\mathrm{CNL}$} \\
\hline 8 & \multicolumn{2}{|c|}{$\mathrm{CNL}$} \\
\hline 9 & \multicolumn{2}{|c|}{$\mathrm{CNL}$} \\
\hline 10 & \multicolumn{2}{|c|}{ CNL } \\
\hline 11 & \multicolumn{2}{|c|}{ CNL } \\
\hline 12 & \multicolumn{2}{|c|}{$\mathrm{CNL}$} \\
\hline 13 & \multicolumn{2}{|c|}{---} \\
\hline 14 & \multicolumn{2}{|c|}{ MSI-H } \\
\hline 15 & \multicolumn{2}{|c|}{ CNL } \\
\hline 16 & \multicolumn{2}{|c|}{$\mathrm{CNL}$} \\
\hline 17 & \multicolumn{2}{|c|}{$\mathrm{CNL}$} \\
\hline 18 & \multicolumn{2}{|c|}{ CNL } \\
\hline
\end{tabular}

CNL, copy number abnormalities low; MSI-H, microsatellite instability hypermutated.

\section{Discussion}

In the present study, we found the differences between histopathological and preoperatively curettage biopsy results in EEC patients, suggesting that treatment decisionmaking based on curettage biopsy results is not reasonable. The other essential finding was that molecular classification could provide vital evidence for prognosis and optimal 
treatment decision-making. Gullo $\mathrm{G}$ et al. reported that progesterone therapy is feasible and effective for young patients with EEC [1]. According to previous studies, successful pregnancy after fertility-sparing treatment in EEC cases has been provided [16]. However, pregnancy failure following fertility-sparing management in patients with EEC remains an unresolved problem and has been rarely reported. Recently, tumor molecular data have been integrated into medical practice decisions, enabling precision medicine [22]. Using molecular categorization to classify the risk of tumors in a fertility-sparing setting has been trialed with promising results [23]. Nevertheless, molecular categorization in such patients, who were diagnosed with EEC and experienced pregnancy failure after fertility-sparing treatment, has been infrequently reported. We hypothesized that molecular classification might provide more reliable prognostic information and guide treatment option decision-making. We herein retrospectively evaluated the molecular features for such EEC patients.

\subsection{Curettage biopsy and histopathological results}

Our results showed heterogeneity in the outcomes of curettage biopsy and postoperative pathology. In the 18 patients of the study, 15 received surgery and underwent endometrial histopathology. We found that 6 patients had differences between preoperative curettage biopsy results and postoperative pathological outcomes; among them, 5 patients were G1 EC in curettage biopsy, but they were G2 EC in postoperative pathological; 1 case was G1 EC in curettage biopsy, but she was malignant Mullerian duct mixed tumor in postoperative pathological. Thus, candidates selection for fertility-sparing treatment only based on curettage biopsy is unreliable, and this viewpoint was supported by several scholars $[3,8,15]$.

\subsection{Molecular classification}

In 2013, The Cancer Genome Atlas (TCGA) had proposed the molecular classification, which classified endometrioid cancers into four subtypes [20]: POLE hypermutation, microsatellite instability (MSI), copy number abnormalities low (CNL), copy number abnormalities high $(\mathrm{CNH})$.

Copy number abnormalities low (CNL) represents that patient with EC have the best prognosis, with an incidence of $39 \%$ [20]. In our study, 15 patients $(15 / 18,83.33 \%)$ with EC were classified as CNL, which usually had no significant molecular characteristics. LeonCastillo et al. reported that the 5-year relapse-free survival rate of patients with CNL type was $74 \%$. Authors also claimed that no significant difference between radiotherapy combined with chemotherapy and radiotherapy alone was found [24]. Therefore, comprehensively evaluating the risk factors, including PCOS syndrome [25, 26], high BMI [9], and tumor family history [2], is essential in EC patients with CNL before fertility-sparing treatment.

POLE hypermutation suggests that patients with EC have a good prognosis, with an incidence of $7 \%$ [20]. In this study, we found that no EEC patients have POLE hypermutation. This result is consistent with the low incidence of POLE hypermutation (7\%). In addition, this may be related to the small sample size of our study.

MSI-H indicates that patients with EC have a moderate prognosis, with an incidence of $28 \%$ [20]. MSI-H has the following characteristics: 1) most EC patients with MSI-H have a pathological type of endometrioid adenocarcinoma; 2) MSI-H is usually detected with mismatched repair genes (MMR) mutations, such as MLH1, MSH2, MSH6, and PMS2, or hypermethylation of the MLH1 gene promoter. In the present study, 2 patients (2/18, $11.11 \%$, cases 2 and 14) had MSI-H. Both patients had a history of pregnancy, normal BMI, regular menstrual cycles, and did not have PCOS syndrome. Case 2 was diagnosed with Lynch syndrome, and she developed colon cancer after EC surgery. Meanwhile, a curettage biopsy of case 14 revealed atypical endometrial hyperplasia and local G1 EC. Case 14 was treated with Mirena, and then curettage biopsy indicated a stable disease. However, postoperative pathological results suggested a more severe G1 EC than preoperatively. Therefore, in our opinion, fertility-sparing treatment is not recommended for patients with MSI-H of molecular classification, especially for patients with Lynch syndrome. 
Copy number abnormalities high $(\mathrm{CNH})$ indicates that EC patients have a poor prognosis, with an incidence of $26 \%$ [20]. In this study, we found that no patients presented with $\mathrm{CNH}$. $\mathrm{CNH}$ incidence in our study was relatively lower than in previous results $(0 \%$ vs. $26 \%$ ). In our opinion, this result was related to the inclusion of subjects because only patients with G1 EC were enrolled, but CNH type is usually found in G3 EC (25\%) and serous adenocarcinoma (75\%). However, gynecologists should consider the misdiagnosis caused by the small tumor tissue materials and inaccurate curettage biopsy results. Consequently, detailed doctor-patient communication is necessary with EC patients who have $\mathrm{CNH}$ and intends to undergo conservative treatment and inform them that the prognosis of fertility-preserving treatment, in this case, is very poor.

In our series, 1 patient (case 13) presented with 'unclassifiable' in molecular classification due to the very low tumor tissue specimen concentration $(<5 \mathrm{ng} / \mathrm{ul})$. Kommoss et al. and his colleagues also found that 3 failures in achieving MMR status were due to the very low tumor volume $(<4 \mathrm{~mm})$, called 'unclassifiable' [27]. In our study, case 13 received medroxyprogesterone acetate tablets for 6 months and showed no tumor recurrence during follow-up. We considered that the patient's good reversal of relapse-free survival was directly related to less tumor tissue.

\subsection{Feasibility and risk of fertility-sparing treatment with progesterone}

Since the treatment of EC with progesterone was first reported in 1961, numerous studies have reported that progesterone treatment of EC is feasible and effective and has achieved a high pregnancy rate and live delivery rate [4, 21, 28, 29]. However, fertility preservation therapy for EC is not a standardized treatment approach [2], and the effective rate of progesterone in treating EC varies greatly among different studies, ranging from $42 \%$ to $88.9 \%$ [30, 31]. Gunderson et al. [32] conducted a meta-analysis of 391 patients with EC who underwent fertility-sparing treatment in 45 studies, with an overall effective rate of $77.7 \%$, the sustained complete remission rate within 39 months of median follow-up was $53.2 \%$, the relapse rate after the initial reaction was $35.4 \%$, and the neoplasm persistence rate was $25.4 \%$. The effect of conservative progesterone therapy on EC is temporary, and nearly half of patients relapse within 3 years of treatment remission. The relapse rate of patients with well-differentiated and EEC treated with progesterone was $23 \% \sim 47 \%$ [ 4 , 21], and a few patients were found to have infiltrated muscle layer when EC recurred[33], or even advanced neoplasm[34]. In addition, some patients died from multiple organ metastasis of neoplasms $[35,36]$. In the present study, the complete remission rate of fertilitysparing treatment was only $16.67 \%$ (3/18), which was relatively lower than previous studies [30-32]. This phenomenon can be interpreted as the inclusion bias in the study because we included women with pregnancy failure after conservative treatment.

\subsection{Treatment following the failure of progesterone therapy}

Progesterone can be used again for patients with relapse and fertility requirements after evaluating tumor grade and myospheric invasion. Park et al. [37] used progesterone for secondary treatment of 33 patients with recurrent EC confined to the uterus, among which 29 patients were treated with MPA $500 \mathrm{mg} / \mathrm{d}$, with a median treatment period of 6 months (3-19 months). They found that 28 patients achieved complete remission, with a complete remission rate of $85 \%$ at a median follow-up of 52 months. In our series, 15 patients with EC relapse, stable, and progression diseases were performed surgical intervention to prevent disease aggravation. Importantly, hysterectomy is the standard treatment approach for stable and progressive diseases after progesterone therapy failure because most lesions were confined to the uterus [38, 39]. Hysterectomy is feasible for patients with relapse and no fertility requirements. Furthermore, staged surgery with attachment preservation or total hysterectomy or adnexectomy only should be performed for patients with failure of progesterone therapy or the disease progression. Besides, staging surgery for endometrial and ovarian cancers combined with chemotherapy or radiation therapy was recommended for patients with adnexal metastasis [24].

\subsection{Risk factors for failure of fertility-sparing treatment}


According to our retrospective analysis of the clinical and pathological features of 18 patients in this study, we found that the reasons for failure of fertility-sparing treatment are as follows: firstly, the patient has various risk factors for EC, such as obesity, PCOS, insulin resistance, diabetes, irregular menstruation, infertility, non-ovulation or sparse ovulation, and amenorrhea; secondly, negative or low expression of PR receptor, or negative or reduced expression of PR receptor during treatment; thirdly, after EC is successfully reversed by fertility-sparing treatment, numerous patients tried natural pregnancy, failed to carry out regular follow-up, and delayed the best period of pregnancy assistance; fourth, after the disease improved effectively by fertility-sparing treatment, some patients presented no continuous regular menstruation, and some patients have amenorrhea, leading to the relapse of the disease; fifth, the lesions infiltrated muscle layer, or the histological grade of the lesions was medium, poorly differentiated or non-endometrioid adenocarcinoma; sixth, patients suffered from other serious medical complications during the process of treatment, such as myocardial infarction, new neoplasms, or a history of other neoplasms, and have to give up treatment failure; seventh, latent ovarian cancer was not found by imaging and laparoscopic exploration. Eighth, the patient did not receive regular treatment due to poor compliance.

\subsection{Feasibility and risk of ovarian preservation in patients who fail fertility-sparing treatment}

In recent years, numerous scholars believed that ovarian preservation did not increase neoplasm-related mortality and did not affect the overall survival rate in young patients with G1 EC [37, 40-42]. Koskas et al. [41] reported 489 patients younger than 40 with G1 intramucosal EC, 101 of them received fertility-sparing treatment, 184 had ovarian preservation, and 204 had both uterus and ovary resection. Multivariate analysis results showed that uterine and ovarian preservation did not increase neoplasm-related mortality or overall survival. In the past 20 years, more than 70 young patients received fertilitysparing treatment for the treatment of atypical endometrial hyperplasia or EEC in our hospital; among them, 15 patients underwent hysterectomy and 12 patients retained ovaries. In the present study, no relapse or death occurred during the median follow-up period of 39 months.

In our opinion, the risk of ovarian preservation in young patients with EEC is longterm ovarian malignancy. Some scholars suggest that laparoscopic lymph node sampling can be performed to detect the presence of extrauterine lesions and ovarian neoplasms [43]. However, even in patients with normal ovarian appearance during intraoperative exploration, there are still $4 \% \sim 25 \%$ patients with latent ovarian malignancy $[4,44]$. Therefore, patients and their families should be fully informed that there is a risk of tumor relapse after surgery.

Although positive outcomes were achieved in the present study, there are still several limitations. Firstly, the limited sample size and retrospective setting represent the main limitations of our preliminary study. The perfect prediction for molecular classification might be due to the high number of covariates compared to the sample size. However, greater sample size and a prospective study design are difficult to obtain in a single center. Therefore, multicenter, prospective, and randomized controlled trials studies should be conducted to evaluate the role of molecular classification in treatment decision-making in EEC patients. Secondly, only CNL and MSI-H types of molecular classification were evaluated because we strictly limited the conditions for inclusion in the study. Thus, POLE and $\mathrm{CNH}$ types need to be further assessed in future studies.

\section{Materials and Methods}

\subsection{Patients}

We enrolled 18 EEC patients who failed fertility-sparing treatment in our hospital from January 2011 to October 2021. The clinical data, pathological features, fertility- 
sparing treatment outcomes, pregnancy outcome, tumor relapse, and prognosis were recorded from medical records, telephone, and outpatient follow-up. The patient's demographic characteristics, including marital status, age at admission, previous pregnancies, body mass index (BMI), cigarette smoke, age at menarche, maximal menstrual cycle length, menstrual phase length, irregular non-menstrual bleeding, irregular menstrual cycle, polycystic ovary syndrome, family history of EC, are listed in Table 1.

Inclusion criteria are as follows: (1) all patients were diagnosed with EEC; (2) the patient was younger than 40 years old; (3) the tumor cell differentiation results of curettage biopsy indicate high differentiation; (4) Imaging results, including MRI or ultrasound, or CT, showed no myometrium infiltration and no distant metastasis of other organs. Exclusion criteria are as follows: (1) the patient was diagnosed with atypical endometrial hyperplasia and other pathological types of EC; (2) the patient was older than 40 years old; (3) absence of written informed consent for the use of biospecimens for research purposes; (4) follow-up period less than 12 months.

\subsection{Fertility-sparing treatment approaches}

(1) High-dose progesterone therapy: Medroxyprogesterone acetate (MPA) (Medroxyprogesterone Acetate Dispersible Tables, Nanjing Xianhe Pharmaceutical Co., Ltd, China), 250-500 mg/d; Or metoprogesterone acetate (MA) (Megestrol Acetate Table, $160 \mathrm{mg} /$ tablet, Bristol Myers Squibb, China), 160-320 mg/d. In the present study, 17 of 18 patients underwent fertility-sparing treatment using high-dose progesterone (Case 1-13, and Case 15-18) (Table 2).

(2) Other treatment methods: it is suitable for patients with obesity, abnormal liver function, and other contraindications of progesterone therapy. Case 14 performed fertility-sparing treatment using levonorgestrel intrauterine sustained release system (LNGIUS) (Mirena, 52mg/piece, Bayer Healthcare Co., LTD. Guangzhou Branch, China), 20 $\mu \mathrm{g} / 24$ hours, because she has the liver disease (Table 2).

\subsection{Evaluation of fertility-sparing treatment outcomes}

Pathologic response to fertility-sparing treatment was categorized as complete remission, stable disease, and progressive disease [21]. Complete remission was defined as the absence of any hyperplastic or cancerous lesion. Stable disease was defined as residual lesion without degeneration or atrophy of endometrial glands. Progressive disease was defined as the appearance of G2 or G3 EC. The progressive disease also was clinically defined when the endometrium showed larger than $20 \mathrm{~mm}$ of the thickness measured by transvaginal ultrasonography or when myometrial invasion or extrauterine lesion were recognized.

All patients underwent curettage biopsy after 3 months of fertility-sparing treatment. If the biopsy result suggests complete remission, the patient stops using high-efficiency progesterone and is prepared for pregnancy. If the biopsy result suggests stable disease, the patient will continue fertility-sparing for the other 3 months until the biopsy results show complete remission. Gynecologists generally recommend high-dose progesterone therapy for less than 6 months [17], to avoid the MPA resistance of EC cells caused by prolonged application of progesterone, resulting in increased proliferation of EC cells. If the biopsy result indicates progressive disease, fertility-sparing was discontinued, and surgery was performed immediately.

\subsection{Surgical treatment strategies}


The same experienced gynecologist performed all operations. Surgical strategies, including laparoscopic hysterectomy (LH), bilateral salpingo-oophorectomy (BSO), transabdominal hysterectomy (TAH), pelvic lymph node dissection (PLND), or bilateral salpingectomy (BS), were performed alone or in combination for the patients who experienced failure of fertility-sparing treatment (Table 2). Before 2016, the criterion of ovariectomy was that the area of lesion in the hysterectomy was greater than $1 / 2$ of the uterine cavity area, and the intraoperative frozen section of the lesions indicated myometrial invasion. After 2016, we performed the intraoperative rapid frozen pathological examination of ovaries before ovariectomy. Then, according to the pathological results, it is decided whether to remove the ovary or not. Moreover, the criterion of PLND was that the area of lesion in the hysterectomy was greater than $1 / 2$ of the uterine cavity area. All patients who received surgical intervention performed a histopathological examination (Table 2).

\subsection{Detection of molecular typing of EC}

We used Next-generation sequencing (NGS) technology for molecular typing detection. The detection platform was Illumina's high-throughput sequencing platform. The samples were achieved by curettage, and prepared into $5 \mu \mathrm{m}$ paraffin sections, 10 pieces, and the tumor cell content was required to be $\geq 30 \%$. The detection included: (1) Exonucase domain of POLE gene (exon 3-14) and a partial region of exon 19; (2) All the coding regions outside of exon 4 of TP53 gene (except partial regions of exon 4), and specific microsatellite (MS). It can detect POLE, TP53, and point mutation, small fragment insertiondeletion, and microsatellite instability state (MSI) in the range of the target region. Besides, the genes, ranges, and types of sequencing are listed in Table 3. The process of molecular typing detection is presented in Figure 1.

\section{Conclusions}

Molecular classification could provide reliable supplementary information for evaluating prognostic and contribute to select treatment strategies in EEC patients. Fertilitysparing treatment is not recommended for EEC patients with $\mathrm{CNH}$ and MSI-H. However, fertility-sparing treatment can be attempted in EEC patients with CHL, and regular follow-up should be carried out to early detection of EC relapse and prevention of disease progression.

Author Contributions: Conceptualization, Xi-Wen Zhang; Data curation, Abdulkarim Mohamed Farah; Investigation, Ping-Li Sun; Supervision, Yan Jia; Writing - original draft, Tong Yu. All authors have read and agreed to the published version of the manuscript.

Funding: Please add: This research received no external funding.

Institutional Review Board Statement: The study was conducted according to the guidelines of the Declaration of Helsinki and approved by the Institutional Review Board of The Second Hospital of Jilin University (In 2021, research review No. 199).

Informed Consent Statement: Informed consent was obtained from all subjects involved in the study.

Data Availability Statement: The datasets used during the current study are available from the corresponding author on reasonable request.

Acknowledgments: None.

Conflicts of Interest: The authors declare no conflict of interest.

\section{References}

1. Gullo, G., A. Etrusco, G. Cucinella, A. Perino, V. Chiantera, A.S. Lagana, et al., Fertility-Sparing Approach in Women 
Affected by Stage I and Low-Grade Endometrial Carcinoma: An Updated Overview. Int J Mol Sci, 2021. 22.

2. Bovicelli, A., G. D'Andrilli, A. Giordano, and P. De Iaco, Conservative treatment of early endometrial cancer. J Cell Physiol, 2013. 228. 1154-1158.

3. Trojano, G., C. Olivieri, R. Tinelli, G.R. Damiani, A. Pellegrino, and E. Cicinelli, Conservative treatment in early stage endometrial cancer: a review. Acta Biomed, 2019. 90. 405-410.

4. Kalogera, E., S.C. Dowdy, and J.N. Bakkum-Gamez, Preserving fertility in young patients with endometrial cancer: current perspectives. Int J Womens Health, 2014. 6. 691-701.

5. Park, H., J.M. Seok, B.S. Yoon, S.J. Seong, J.Y. Kim, J.Y. Shim, et al., Effectiveness of high-dose progestin and long-term outcomes in young women with early-stage, well-differentiated endometrioid adenocarcinoma of uterine endometrium. Arch Gynecol Obstet, 2012. 285. 473-478.

6. Galczynski, K., L. Nowakowski, T. Rechberger, and A. Semczuk, Should we be more aware of endometrial cancer in adolescents? Dev Period Med, 2016. 20. 169-173.

7. Kim, S.M., S.J. Shin, J.G. Bae, K.Y. Kwon, and J.H. Rhee, Endometrial adenocarcinoma in a 13-year-old girl. Obstet Gynecol Sci, 2016. 59. 152-156.

8. Corzo, C., N. Barrientos Santillan, S.N. Westin, and P.T. Ramirez, Updates on Conservative Management of Endometrial Cancer. J Minim Invasive Gynecol, 2018. 25. 308-313.

9. La Russa, M., I. Zapardiel, M.J. Halaska, K. Zalewski, R. Laky, P. Dursun, et al., Conservative management of endometrial cancer: a survey amongst European clinicians. Arch Gynecol Obstet, 2018. 298. 373-380.

10. Giampaolino, P., A. Di Spiezio Sardo, A. Mollo, A. Raffone, A. Travaglino, A. Boccellino, et al., Hysteroscopic Endometrial Focal Resection followed by Levonorgestrel Intrauterine Device Insertion as a Fertility-Sparing Treatment of Atypical Endometrial Hyperplasia and Early Endometrial Cancer: A Retrospective Study. J Minim Invasive Gynecol, 2019. 26. 648-656.

11. La Rosa, V.L., S. Garzon, G. Gullo, M. Fichera, G. Sisti, P. Gallo, et al., Fertility preservation in women affected by gynaecological cancer: the importance of an integrated gynaecological and psychological approach. Ecancermedicalscience, 2020. 14. 1035.

12. Baker, J., A. Obermair, V. Gebski, and M. Janda, Efficacy of oral or intrauterine device-delivered progestin in patients with complex endometrial hyperplasia with atypia or early endometrial adenocarcinoma: a meta-analysis and systematic review of the literature. Gynecol Oncol, 2012. 125. 263-270.

13. Chandra, V., J.J. Kim, D.M. Benbrook, A. Dwivedi, and R. Rai, Therapeutic options for management of endometrial hyperplasia. J Gynecol Oncol, 2016. 27. e8.

14. Kim, M.K., S.J. Seong, J.W. Kim, S. Jeon, H.S. Choi, I.H. Lee, et al., Management of Endometrial Hyperplasia With a Levonorgestrel-Releasing Intrauterine System: A Korean Gynecologic-Oncology Group Study. Int J Gynecol Cancer, 2016. 26. 711-715.

15. Yang, B., Y. Xu, Q. Zhu, L. Xie, W. Shan, C. Ning, et al., Treatment efficiency of comprehensive hysteroscopic evaluation and lesion resection combined with progestin therapy in young women with endometrial atypical hyperplasia and endometrial cancer. Gynecol Oncol, 2019. 153. 55-62.

16. Novikova, O.V., I. Andreeva, E.G. Novikova, A.N. Vostrov, S.M. Pronin, N.A. Rubtsova, et al., Hormone therapy alone for pre-cancerous conditions and early endometrial cancer: pros and cons. Vopr Onkol, 2014. 60. 306-312.

17. Garg, K. and R.A. Soslow, Endometrial carcinoma in women aged 40 years and younger. Arch Pathol Lab Med, 2014. 138. 335-342.

18. Bogani, G., S.C. Dowdy, W.A. Cliby, F. Ghezzi, D. Rossetti, L. Frigerio, et al., Management of endometrial cancer: issues and controversies. Eur J Gynaecol Oncol, 2016. 37. 6-12.

19. Caponio, M.A., T. Addati, O. Popescu, S. Petroni, V. Rubini, M. Centrone, et al., P16(INK4a) protein expression in 
endocervical, endometrial and metastatic adenocarcinomas of extra-uterine origin: diagnostic and clinical considerations. Cancer Biomark, 2014. 14. 169-175.

20. Cancer Genome Atlas Research, N., C. Kandoth, N. Schultz, A.D. Cherniack, R. Akbani, Y. Liu, et al., Integrated genomic characterization of endometrial carcinoma. Nature, 2013. 497. 67-73.

21. Ushijima, K., H. Yahata, H. Yoshikawa, I. Konishi, T. Yasugi, T. Saito, et al., Multicenter phase II study of fertility-sparing treatment with medroxyprogesterone acetate for endometrial carcinoma and atypical hyperplasia in young women. J Clin Oncol, 2007. 25. 2798-2803.

22. Li, M.M., M. Datto, E.J. Duncavage, S. Kulkarni, N.I. Lindeman, S. Roy, et al., Standards and Guidelines for the Interpretation and Reporting of Sequence Variants in Cancer: A Joint Consensus Recommendation of the Association for Molecular Pathology, American Society of Clinical Oncology, and College of American Pathologists. J Mol Diagn, 2017. 19. 4-23.

23. Falcone, F., N. Normanno, N.S. Losito, G. Scognamiglio, R. Esposito Abate, N. Chicchinelli, et al., Application of the Proactive Molecular Risk Classifier for Endometrial Cancer (ProMisE) to patients conservatively treated: Outcomes from an institutional series. Eur J Obstet Gynecol Reprod Biol, 2019. 240. 220-225.

24. Leon-Castillo, A., S.M. de Boer, M.E. Powell, L.R. Mileshkin, H.J. Mackay, A. Leary, et al., Molecular Classification of the PORTEC-3 Trial for High-Risk Endometrial Cancer: Impact on Prognosis and Benefit From Adjuvant Therapy. J Clin Oncol, 2020. 38. 3388-3397.

25. Lagana, A.S., P. Rossetti, M. Buscema, S. La Vignera, R.A. Condorelli, G. Gullo, et al., Metabolism and Ovarian Function in PCOS Women: A Therapeutic Approach with Inositols. Int J Endocrinol, 2016. 2016. 6306410.

26. Bezerra Espinola, M.S., A.S. Lagana, G. Bilotta, G. Gullo, C. Aragona, and V. Unfer, D-chiro-inositol Induces Ovulation in Non-Polycystic Ovary Syndrome (PCOS), Non-Insulin-Resistant Young Women, Likely by Modulating Aromatase Expression: A Report of 2 Cases. Am J Case Rep, 2021. 22. e932722.

27. Kommoss, S., M.K. McConechy, F. Kommoss, S. Leung, A. Bunz, J. Magrill, et al., Final validation of the ProMisE molecular classifier for endometrial carcinoma in a large population-based case series. Annals of Oncology, 2018. 29. 1180-1188.

28. Dursun, P., S. Erkanli, A.B. Guzel, M. Gultekin, N.C. Tarhan, O. Altundag, et al., A Turkish Gynecologic Oncology Group study of fertility-sparing treatment for early-stage endometrial cancer. Int J Gynaecol Obstet, 2012. 119. 270-273.

29. Jadoul, P. and J. Donnez, Conservative treatment may be beneficial for young women with atypical endometrial hyperplasia or endometrial adenocarcinoma. Fertil Steril, 2003. 80. 1315-1324.

30. Koskas, M., J. Uzan, D. Luton, R. Rouzier, and E. Darai, Prognostic factors of oncologic and reproductive outcomes in fertility-sparing management of endometrial atypical hyperplasia and adenocarcinoma: systematic review and metaanalysis. Fertil Steril, 2014. 101. 785-794.

31. Gallos, I.D., J. Yap, M. Rajkhowa, D.M. Luesley, A. Coomarasamy, and J.K. Gupta, Regression, relapse, and live birth rates with fertility-sparing therapy for endometrial cancer and atypical complex endometrial hyperplasia: a systematic review and metaanalysis. Am J Obstet Gynecol, 2012. 207. 266 e261-212.

32. Gunderson, C.C., A.N. Fader, K.A. Carson, and R.E. Bristow, Oncologic and reproductive outcomes with progestin therapy in women with endometrial hyperplasia and grade 1 adenocarcinoma: a systematic review. Gynecol Oncol, 2012. 125. 477482.

33. Hurst, S.A., K.M. Hartzfeld, and G. Del Priore, Occult myometrial recurrence after progesterone therapy to preserve fertility in a young patient with endometrial cancer. Fertil Steril, 2008. 89. 724 e721-723.

34. Kothari, R., L. Seamon, D. Cohn, J. Fowler, and D.M. O'Malley, Stage IV endometrial cancer after failed conservative management: a case report. Gynecol Oncol, 2008. 111. 579-582.

35. Cormio, G., R. Martino, V. Loizzi, L. Resta, and L. Selvaggi, A rare case of choroidal metastasis presented after conservative management of endometrial cancer. Int J Gynecol Cancer, 2006. 16. 2044-2048. 
36. Huang, S.Y., S.M. Jung, K.K. Ng, Y.C. Chang, and C.H. Lai, Ovarian metastasis in a nulliparous woman with endometrial adenocarcinoma failing conservative hormonal treatment. Gynecol Oncol, 2005. 97. 652-655.

37. Park, J.Y., S.H. Lee, S.J. Seong, D.Y. Kim, T.J. Kim, J.W. Kim, et al., Progestin re-treatment in patients with recurrent endometrial adenocarcinoma after successful fertility-sparing management using progestin. Gynecol Oncol, 2013. 129. 7-11.

38. Passarello, K., S. Kurian, and V. Villanueva, Endometrial Cancer: An Overview of Pathophysiology, Management, and Care. Semin Oncol Nurs, 2019. 35. 157-165.

39. Abu-Rustum, N.R., C.M. Yashar, K. Bradley, S.M. Campos, J. Chino, H.S. Chon, et al., NCCN Guidelines(R) Insights: Uterine Neoplasms, Version 3.2021. J Natl Compr Canc Netw, 2021. 19. 888-895.

40. Sun, C., G. Chen, Z. Yang, J. Jiang, X. Yang, N. Li, et al., Safety of ovarian preservation in young patients with early-stage endometrial cancer: a retrospective study and meta-analysis. Fertil Steril, 2013. 100. 782-787.

41. Koskas, M., S. Bendifallah, D. Luton, E. Darai, and R. Rouzier, Safety of uterine and/or ovarian preservation in young women with grade 1 intramucous endometrial adenocarcinoma: a comparison of survival according to the extent of surgery. Fertil Steril, 2012. 98. 1229-1235.

42. Wright, J.D., A.M. Buck, M. Shah, W.M. Burke, P.B. Schiff, and T.J. Herzog, Safety of ovarian preservation in premenopausal women with endometrial cancer. J Clin Oncol, 2009. 27. 1214-1219.

43. Navarria, I., M. Usel, E. Rapiti, I. Neyroud-Caspar, M.F. Pelte, C. Bouchardy, et al., Young patients with endometrial cancer: how many could be eligible for fertility-sparing treatment? Gynecol Oncol, 2009. 114. 448-451.

44. Walsh, C., C. Holschneider, Y. Hoang, K. Tieu, B. Karlan, and I. Cass, Coexisting ovarian malignancy in young women with endometrial cancer. Obstet Gynecol, 2005. 106. 693-699. 